\title{
Electrocardiogram Events Detection
}

\author{
João Paulo Teixeira ${ }^{1}$ and Vanda Lopes ${ }^{2}$ \\ ${ }^{1}$ Polytechnic Institute of Bragança, Portugal, joaopt@ipb.pt, \\ ${ }^{2}$ University of Minho, Braga, Portugal, vandacarolina@hotmail.com
}

\begin{abstract}
This work aims to create a system of medical diagnosis of the Electrocardiogram (ECG). The events of the ECG are related with the functioning of the heart and different disorders of the heart functioning have their own ECG pattern allowing the connection between ECG patterns and cardiac disorders. For this purpose, we present here an algorithm that detects the P, QRS and T events of the ECG under MATLAB environment. The algorithm is based in two techniques. The search for picks and valleys and the search of event using a known patter and the correlation with ECG signal inside of a previously detected period. Finally the results are presented and discussed.
\end{abstract}

Keywords: ECG signal, P QRS and T events detection.

\section{Introduction}

The purpose of present project relays on a help system for medical diagnose of cardiac diseases using ECG exam. An algorithm to automatically detect the P, QRS and $\mathrm{T}$ events is presented here. The measure of the hit rate is also discussed.

The algorithm identifies the QRS points and the beginning, end and the maximum points of the $\mathrm{P}$ and $\mathrm{T}$ events.

The malfunction of the cardiac cycle due to changes in the heart morphology will modify the propagation of the repolarisation / depolarasation waves. Each heart disease makes standard changes in the signal of electrocardiogram. The changes mostly consist in missed events, ECG modifications and shortening or lengthening the ECG periods. Therefore, it is possible to connect the ECG signal and cardiac diseases.

\subsection{The Heart}

The heart is the most important organ of the human body. This organ is a central pump and has the mission of create blood pressure that provides oxygen and nutrients to cells.

The heart is a muscular organ the size of a fist with four chambers: right atrium (or auricle), left atrium, right ventricle and left ventricle [1], [2]. The two chambers upper and lower are called auricles and ventricles respectively. The wall that separates the heart into a right and left side are called septum [2]. The two sides of the heart are like a twin pumps. They are combined in a single organ but placed in series in the vascular system, where their connections have the purpose of separating the arterial from 
venous blood. The arterial blood is rich in oxygen and the venous blood in carbon dioxide [1].

To direct the flow of blood and prevent its backward movement the heart has four valves. The valves between the auricles and the ventricles are atrioventricular valves. The atrioventricular valve on the left side is called bicuspid or mitral and the atrioventricular valve on the right side is called tricuspid. Another two valves are the semilunar valves and they are between the ventricles and their attached vessels [2].

\subsection{Cardiac Cycle}

The human heart beats ceaselessly about 70 times a minute. The cardiac cycle begins with the simultaneous contraction of the auricles. Then, same happens with ventricles and the auricles and the ventricles relax. The phase of contraction the chambers is called systole and the phase of relax is called diastole. When the auricles are in diastole, the ventricles are in systole and vice versa.

When the auricles are in systole, the atrioventricular valves are open and the semilunar valves are open when the ventricles are in systole [2].

\subsection{Control of Heartbeat}

The intrinsic conduction system is responsible to the rhythmical contraction of the heart. There is a type of a cardiac muscle located in two regions of the heart. This muscle has muscular and nervous characteristics and is called nodal tissue. The two nodes in the heart are the sinoatrial node (or SA node) and the atrioventricular node (or AV node). The SA node is located in the upper dorsal wall of the right auricle and the AV node is located in the base of the right auricle very near the septum [1], [2].

The heart beat initiate with an exciting impulse sent by the sinoatrial node. This impulse causes the contraction of the auricles. The impulse reaches the atrioventricular node and this cause a slight delay allowing the auricles finish the contraction. The next step is the contraction of the ventricles. The impulse is now sent to the atrioventricular bundle (or AV bundle) and then immediately arrives to the numerous and smaller Purkinje fibers [2].

As explained, the sinoatrial node is very important because his responsible to keep the heartbeat regular. If the SA node fails the heart still beats by the impulses generated by the AV node. However, the rhythm is slower [2].

\subsection{The Electrocardiogram}

The electrical changes that occur during a cardiac cycle can be recorded by an ECG. The electrical impulse that travels through the heart is conducted by the ions present in the body fluids. These ions contained in the body fluids allow electrical changes in the cardiac cycle can be detected on the skin's surface [2]. 
To take a electrocardiogram exam it's necessary connect electrodes placed on the skin to an instrument (electrocardiograph) that detects the electrical changes in the heart.

When the sinoatrial node sends an impulse the atrial fibers generate an electrical change that is called the $\mathrm{P}$ wave, as depicted in Fig. 1. This wave provides information that the auricle are about to contract. When the ventricles are about to contract, we obtain the QRS complex. Finally, the relaxation of the ventricles is recorded and produces the $\mathrm{T}$ wave.

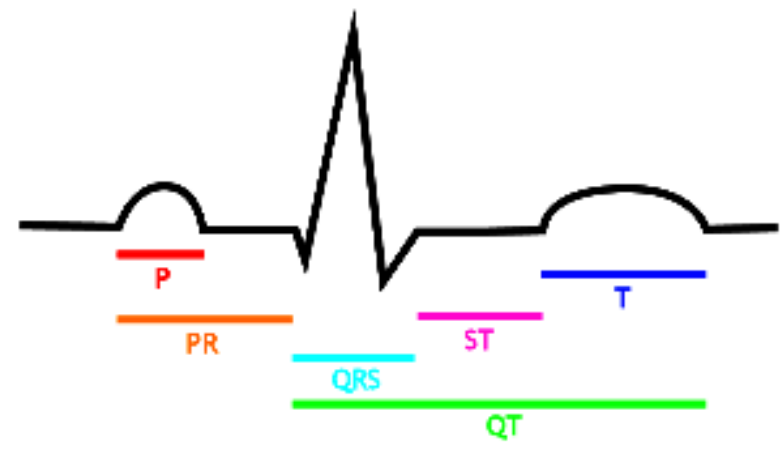

Fig. 1. Events of the electrocardiogram.

\section{Automatic Detection of Electrocardiogram Events}

To rich the automatic system to diagnose cardiac diseases based on the ECG, the first step consist in developing an algorithm to automatically detect the events in a normal ECG.

For that purpose we are developing our algorithm under Matlab environment. The ECG signals used here were downloaded from the database PhysioBank-physiologic signal archives for biomedical research [3], and consist in sello0m.mat and 16265 m.mat.

The signal sel100m.mat has a sampling frequency of $250 \mathrm{~Hz}$ and was used to detect the normal electrocardiogram events. The 16265m.mat signal has a sampling frequency of $128 \mathrm{~Hz}$ and as used to develop the algorithm and was also used to measure the hit rate performance of the algorithm.

\subsection{Signal Preprocessing}

The signal extracted from the database has duration of 12 hours. To not have to work with such a huge number of samples (1000000 samples), we used only one section of it with 10000 samples (Fig. 2). After loading the signal, we obtained the section as follows: 


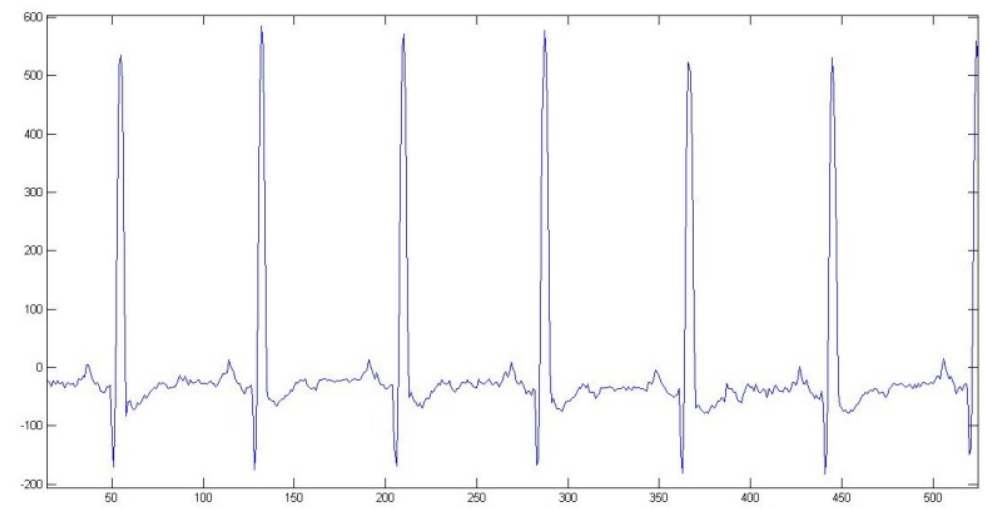

Fig. 2. Section of the first 520 samples of the signal 16265m.mat.

The ECG signal extracted on the surface of the skin's by the ECG has some oscillation due to electrical noise, small movements of the body and a contact not always perfect between the skin and the electrode. The noise in the signal is originated by the functioning of others organs and makes it harder to identify the electrocardiogram events. This oscillation consists in high frequency components of low energy producing small oscillations in the ECG signal. Thus, first at all the signal must be smoothed. For this, we used the moving average filter and a detrend filter. The smoothing process is very critical to remove all the picks and valleys others than the expected P, QRS and T waves once the algorithm uses the search of picks and valleys starting from a known point.

The moving average filter consists in to recalculate the value of an experimental measurement using the average of points ahead and behind that measure. It's designated as a window length, $\mathrm{N}$, the number of used points.

In the implementation of this filter it was necessary to determine the length of the section of signal used L using the command length and the central point of the window $\mathrm{N}$, which is rounded to integer $\mathrm{M}$ using the floor command., as illustrated in next command lines.

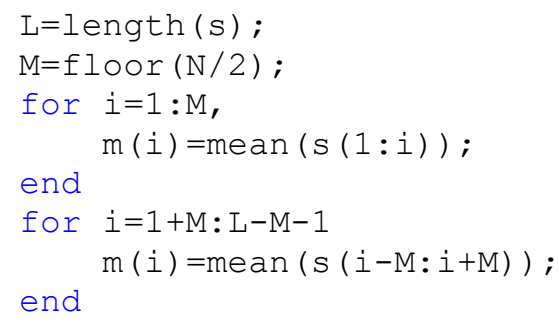




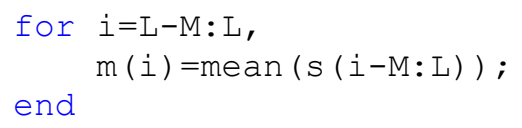

The first and last for cycles carry on the moving average in the beginning and end of the signal where no $\mathrm{M}$ points are available for the moving average. The second for cycle implements de moving average over the middle points of the signal.

The detrend filter consists to remove a possible linear trend using the Fast Fourier Transform (FFT) processing [4].

In our signal we start smoothing the ECG signal with a moving average filter with length $\mathrm{N}=11$, and using the detrend filter. An example of the smoothed signal is presented in Fig. 3.

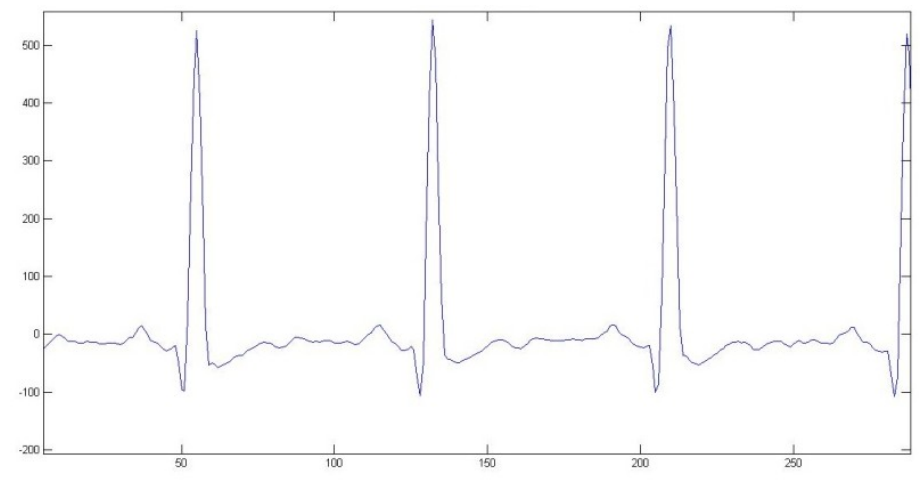

Fig. 3. Smoothed signal.

\subsection{Automatic Determination of Periods}

The duration of cardiac cycle varies within patients and for the same patient it may have soft variations or even strong variations depending on the disease. The cardiac cycle can be measured as the duration between consecutive QRS complex.

To identify the duration of the cardiac cycle we used a technique based in the correlation coefficient. The correlation coefficient is the measure of a relationship between two vectors, this coefficient indicates the direction and strength between them [5].

The technique consists on the use of a vector of samples with the shape we are looking for in the signal and determine the successive correlation coefficients along the signal. This process results in a vector with the same length of the signal and with values very close to one in the positions in the signal with the searched shape.

The event, which was used for automatic detection of periods, was the vector corresponding to the QRS complex. We start by finding the maximum value and the rate at which this vector occur. Then a normalization of the amplitude took place. For points between the start signal and end signal subtracted from the vector length of less 
than one comparison was also carried out a normalization of the amplitude. Finally, the correlation was determined:

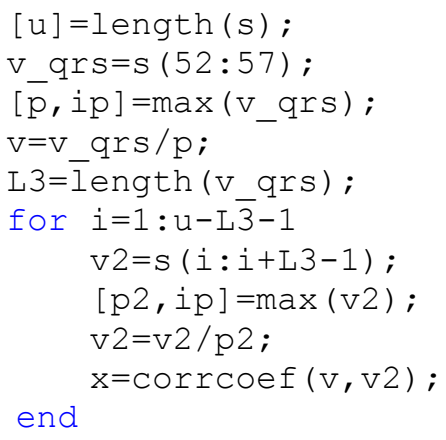

Figure 4 shows the signal and below the graph of the correlation. In this graph we can see that the values of correlation are between -1 and 1 . We also found that when we are at the beginning of the QRS complex (Q point), the correlation is equal to 1 , but with the increase of half the length of the vector of comparison gives the central position of the QRS complex.

The aim is to record the values of the indices in which there is a QRS complex in the vector.

As in several positions of the signal, the correlation is too high (close to one) and other situations may not even be equal to 1 , the calculation of periods made itself a condition for the search of the QRS complex. Then the program only records the value of the correlation higher than 0.98 . This value was experimentally obtained through the analysis of correlation graphs. Finally, the calculation of the period of the first cycle is performed.

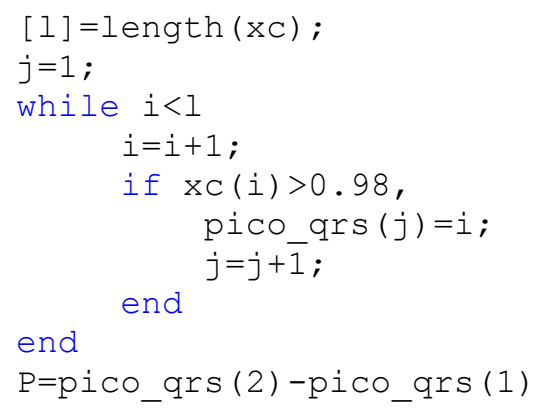

The $\mathrm{xC}$ vector is the a segment of the correlation vector $\mathrm{x}$ where the QRS complex is expected as possible.

This method is very sensitive to the size of the vector used for the comparison, because if this is small will often be found along the same cycle and if you have a longer length will not be found in all cycles. 


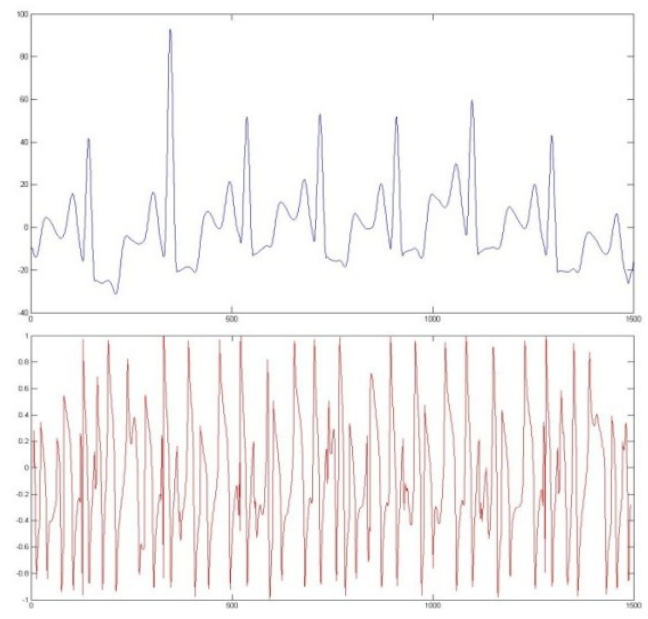

Fig. 4. Correlation of the QRS complex.

\subsection{Detection of ECG Events}

The identification of the ECG events has based in the correlation coefficient technique, described before, and in the determination of picks and valleys.

The search of events has made using a segment of the original signal containing one period, plus about $20 \%$ of a period samples before and after. The periods were determined as described before. Therefore the algorithm described in this section is repeats along the periods of the ECG.

The algorithm began by creating a variable $j$ which would be a sample reference inside the complete ECG signal. It is repositioned for a number of samples for the cycle being entirely represented. Once created a condition in which the detection of events occurring up to be an initial number to ensure that does not begin a cycle that is not full. The initial number was 170 samples.

We begin by numbering each cycle before the different complexes. We used a variable named cycle and were initiated at 0 . Next, we selected a segment (s3) to search for the signal events of the ECG. This segment begins in the $j$ position and has the length of the period plus $20 \%$.

The next step was to detect the cycle period in which we were to experience the events of the ECG. For this, we seek the maximum value and its index in s3. If it is the first cycle, then the period is determined by the difference to the beginning of first QRS complex (144). However, if it is not the first cycle then the period shall be the index of maximum amplitude plus 50 (in order to guarantee one complete period). The end of the period will be recalculated later. The period, in seconds, is determined dividing the number of samples by the sampling frequency $(\mathrm{Fa})$.

$$
\text { [pico, indp] }=\max (\text { s3) ; }
$$




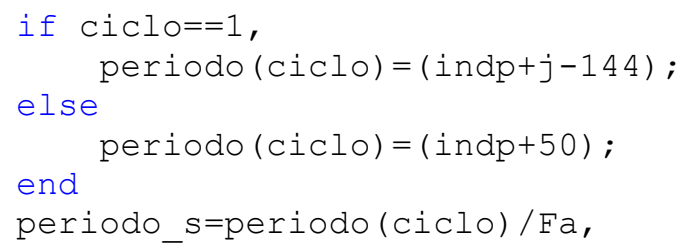

The first event to be detected was the point $\mathrm{R}$. This point is exactly the maximum point previously calculated. Next step consist in adjust the end of our search area added to the index $j$ to the R-point approximation of $90 \%$ over the period.

Behind the point $\mathrm{R}$ is the position of the $\mathrm{P}$ wave, more precisely the end of $\mathrm{P}$ wave. The only way found to make its detection was by correlation technique. It was carried out in exactly the same way as explained earlier, using and adequate vector for comparison. Within the specified condition we just have give the legal position for the end of $\mathrm{P}$ point, that is between the start of the cardiac cycle and the point $\mathrm{R}$. We have to add the variable $j$, because this variable is the position inside the entire signal.

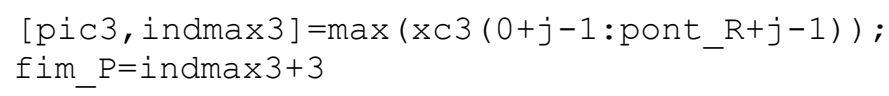

The correlation procedure was also used to find the Q point, and the search area (legal zone) goes from the end of $\mathrm{P}$ wave to the $\mathrm{R}$ point. The point that marks the beginning of the $\mathrm{T}$ wave has also found by the correlation procedure with the legal zone beginning at the $\mathrm{S}$ point to the result of the value of the period plus a number that allows us to look without that section of the signal ends.

For the other ECG events, the procedure was the search of picks and valleys.

Therefore, we can detect an event through the end of an ascent or a descent. Thus, with reference to the end of the $\mathrm{P}$ wave signal backwards we found a rise and at the end of the rise is the maximum of the $\mathrm{P}$ wave.

On the other way if the point is higher than previous one, and this was repeated some points, we are witnessing a rise. Obviously one begins to decrement in the section on the end of the $\mathrm{P}$ wave.

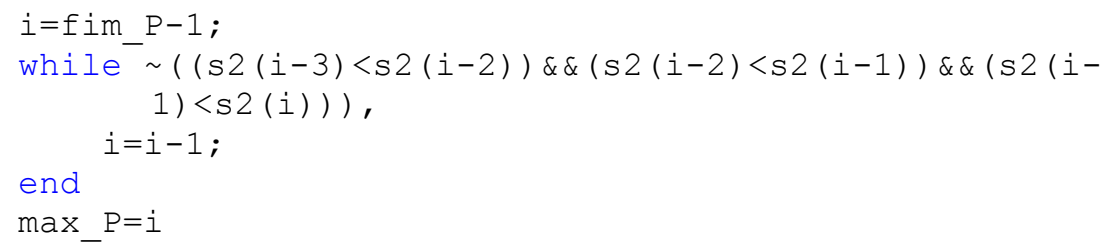

This method of ascent is also used to detect the maximum of the T wave. To do this we must move forward from the point $\mathrm{R}$ and started looking for him after the start of the $\mathrm{T}$ wave. But in place of decrements will be increments.

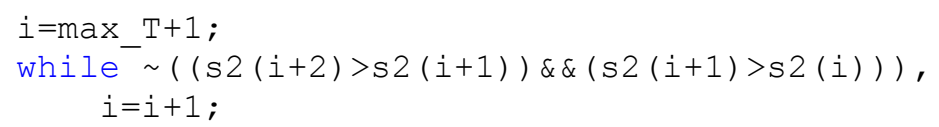


end

fim_ $\mathrm{T}=\mathrm{i}$;

The beginning of the $\mathrm{P}$ wave was determined by searching for the valley on the left of the maximum of $\mathrm{P}$ wave.

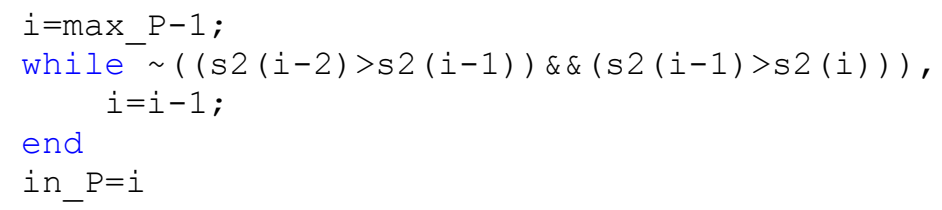

This procedure is used to detect the $\mathrm{S}$ and the end of the $\mathrm{T}$ wave, with the difference that we start from $\mathrm{R}$ point and maximum $\mathrm{T}$, respectively.

To advance to the next cycle, only $j$ will assume the value of previous $j$ plus the $R$ point and added to a safety margin of 50 samples.

\section{Results}

In fig. 5 all points of interest marked in a single cycle are displayed. In order to measure the efficiency of the algorithm, the hit rate for the all events and points, given by eq. 2 , was determined over a 100 cardiac cycles. In table 1 , we can see the hit rate for each point of the ECG.

$$
\text { Hit Rate }=\text { number of hits } / \text { number of periods }
$$

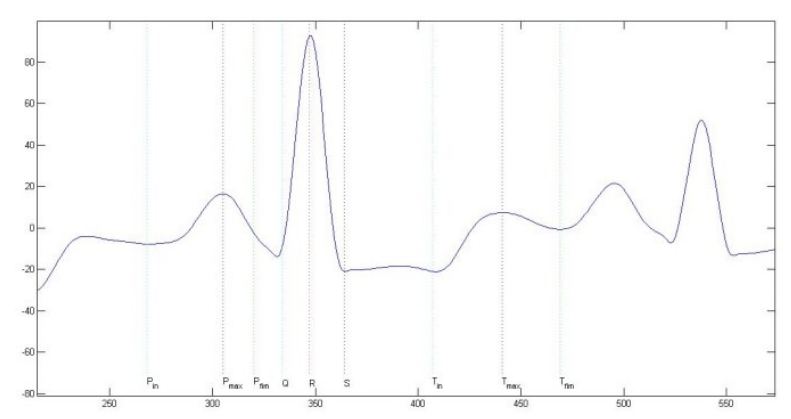

Fig. 5. One cycle of the ECG events.

Table 1- Hit rate in \%, for each point of the ECG

\begin{tabular}{|cccccccccc|}
\hline Point & $\begin{array}{c}\text { Begin. } \\
\text { of } \mathbf{P}\end{array}$ & $\begin{array}{c}\text { Max } \\
\mathbf{P}\end{array}$ & $\begin{array}{c}\text { End } \\
\text { of P }\end{array}$ & $\begin{array}{c}\text { Q } \\
\text { Point }\end{array}$ & $\begin{array}{c}\text { R } \\
\text { Point }\end{array}$ & $\begin{array}{c}\text { S } \\
\text { Point }\end{array}$ & $\begin{array}{c}\text { Begin. } \\
\text { of T }\end{array}$ & $\begin{array}{c}\text { Max } \\
\text { T }\end{array}$ & $\begin{array}{c}\text { End } \\
\text { of T }\end{array}$ \\
$\begin{array}{c}\text { Hit } \\
\text { Rate }\end{array}$ & 56 & 56 & 56 & 66 & 90 & 90 & 41 & 36 & 36 \\
\hline
\end{tabular}


These hit rates can be improved with better filtering of noise, because in some cardiac cycles smoothing was high, which complicates the detection of some points of the ECG, including the $\mathrm{P}$ and $\mathrm{T}$ wave.

\section{Conclusions}

An algorithm to determine the events of a normal ECG is presented as a part of a medical system for suggest the diagnosis of cardiac diseases over the ECG.

The algorithm start by filtering the ECG signal in order to smooth the signal using a moving average and a detrend filters. This smoothing process is very sensitive to the performance of the following algorithm, because of the picks and valleys technique used for search of several point.

Than the periods has determined by searching the QRS complex using a new proposed technique based in the correlation coefficient.

The reference point was the point $\mathrm{R}$, from this point was determined the $\mathrm{Q}$ point and the end of $\mathrm{P}$ wave. Through the end of $\mathrm{P}$ wave we find the maximum point and then the beginning of the $\mathrm{P}$ wave. These points were calculated by searching the picks and valleys. The following points were determined also by the search of picks and valleys and were determined in the following order: $\mathrm{S}$ point, the beginning, the maximum and the end of the $\mathrm{T}$ wave.

This algorithm can give good results if the smoothing process is adequate, as presented in fig. 5, but final results presented in table 1 showed that more robust method should be used or the smoothing process should be improved.

In the future a wavelet technique to search the events should be implemented.

\section{References}

1. Gray, Henry, Williams, Peter Llewellyn and Bannister, Lawrence H.: Gray's Anatomy. $38^{\circ}$. Churchill Livingstone (1995)

2. Mader, Sylvia S.: Human Biology. McGraw-Hill (2006)

3. Bioengineering, National Institute of Biomedical Imaging and PhysioBank, http://www.physionet.org/physiobank/about.shtml

4. Yang, Won Y.: Signals and Systems with MATLAB. Springer, (2009)

5. Guimarães, Rui Campos and Cabral, Jose A. Sarsfield: Estatística. Mcgraw-hill (1997)

6. Moreira, Adelino Leite and Chaves, Paulo Castro: Electrocardiografia. Porto: Faculdade de Medicina da Universidade do Porto - Serviço de Fisiologia (2001)

7. Silverthorn, Dee Unglaub, William C. Ober: Human Physiology: An Integrated Approach with Interactive Physiology. 4th ed., San Francisco (2009)

8. Guyton, Arthur C.: Tratado de Fisiologia Médica. 9a: Guanabara Koogan (1996)

9. Davis, Goode P. and Parks, Edward: O corpo humano . Alfragide: Ediclube. Vol. The Heart. The Living Pump (1987)

10. Feldman, José and Goldwasser, Gerson P.: Electrocardiograma: Recomendações para a sua interpretação. Universidade Federal do Rio de Janeiro, Faculdade de Medicina Souza Marques, Universidade Gama Filho (2004) 